\title{
Análise das Propostas de Inovação nos Trabalhos de Conclusão de Curso de um Programa de Mestrado Profissional em Matemática
}

\author{
Adriana Breda, Universidad de Los Lagos, (Chile) \\ Vicenç Font, Universitat de Barcelona, (Espanha) \\ Valderez Marina do Rosário Lima, Pontifícia Universidade Católica do Rio Grande do \\ Sul, (Brasil)
}

Recibido el 21 de marzo de 2016; aceptado el 14 de julio de 2016

\begin{abstract}
Análise das propostas de inovação nos trabalhos de conclusão de curso de um programa de mestrado profissional em matemática

\section{Resumo}

Este trabalho tem como objetivo geral determinar quais são, e como se justificam, as inovações nos trabalhos de conclusão de curso do Mestrado Profissional em Matemática em Rede Nacional (PROFMAT). Para isso, realizou-se um estudo qualitativo de vinte e nove trabalhos publicados nos anos de 2013 e 2014. Conclui-se que os dois principais tipos de inovação são a incorporação de conteúdos de nível superior na Educação Básica e a introdução de recursos informáticos. O primeiro tipo se justifica porque permite novas maneiras de relacionar e abordar conteúdos matemáticos, a realização de processos matemáticos relevantes e aulas mais atrativas que despertam o interesse dos alunos. $\mathrm{O}$ segundo tipo se justifica porque as TIC estimulam, motivam, tornam as aulas mais incitantes e são ferramentas que facilitam a abordagem de conceitos e a resolução de problemas.
\end{abstract}

Palavras-chave: Trabalho de conclusão de curso, Mestrado Profissional em Matemática, Novos conteúdos matemáticos, TIC.

Análisis de propuestas de innovación en los trabajos finales de un programa de Máster Profesional en Matemáticas

\section{Resumen}

Este trabajo tiene como objetivo general determinar cuáles son y cómo se justifican, las innovaciones presentadas en los trabajos de fin de máster de la Maestría Profesional en Matemáticas en la Red Nacional (PROFMAT). Para ello, se realizó un estudio cualitativo de veintinueve trabajos de fin de máster publicados en los años 2013 y 2014. Se concluye que los dos tipos principales de innovación son la incorporación de contenidos de matemáticas de nivel superior en la Educación Básica y la introducción de los recursos informáticos. El primer tipo se justifica porque permite nuevas formas de relacionar y abordar los contenidos matemáticos, la realización de procesos matemáticos relevantes e impartir clases más atractivas que despiertan el interés de los estudiantes. El segundo tipo se justifica porque las TIC estimulan, motivan, hacen las clases más interesantes y son herramientas que facilitan la comprensión de conceptos y la resolución de problemas.

Para citar: Breda, A.; Font, V \& Lima, V. M. (2016). Análise das Propostas de Inovação nos Trabalhos de Conclusão de Curso de um Programa de Mestrado Profissional em Matemática. Avances de Investigación en Educación Matemática, 10, 53-72 
Palabras clave: Trabajo de fin de máster, Máster Profesional en Matemáticas, nuevos contenidos matemáticos, TIC.

\section{Analysis of innovation proposals from the final course assignments in a professional master program in mathematics}

\section{Abstract}

The main objective of this work is finding out the innovations and their justifications within the Brazilian Professional Master Program in Mathematics (PROFMAT). A qualitative study is carried out by addressing a total of 29 works published from the first half of 2013 to the second half of 2014. It is concluded that the main kinds of innovation are: (i) incorporation of higher education content on basic education, and (ii) introduction of computational resources. The former is justified because it allows new ways of relating and addressing mathematical content, conducting relevant mathematical processes and doing more attractive the lessons to stimulate the students' interest. The latter is justified by the fact that ICT motivates and stimulate lessons that contribute to facilitate conceptual understanding and problem solving.

Keywords: Final course assignment, Professional Master in Mathematics, New mathematical content, ICT.

Analyse des propositions d'innovation les innovations présentées dans les mémoires finaux de master professionnel en mathématiques

\section{Résumé}

Ce travail de recherche a pour objectif général de déterminer quelles sont (et comment on les justifie) les innovations présentées dans les mémoires finaux de Master Professionnel en Mathématiques du Réseau National (PROFMAT). Pour ce faire, on a effectué une étude qualitative de vingt-neuf travaux de fin de master publiés en 2013 et 2014. On en conclut que les deux lignes principales d'innovation se rapportent à l'intégration de contenus de mathématiques de niveau supérieur aux programmes de l'Enseignement Obligatoire et à l'introduction de ressources informatiques. La première ligne se justifie parce qu'elle permet de nouvelles manières de mettre en relation et d'aborder les contenus mathématiques, la concrétisation de processus mathématiques remarquables et de donner des cours plus attrayants de façon à éveiller l'intérêt des élèves. La deuxième innovation se justifie parce que les TIC stimulent, encouragent et rendent les cours plus intéressants. Il s'agit d'outils qui facilitent la compréhension de concepts et la solution de problèmes.

Mots clés: Mémoire de fin de master, Master Professionnel en Mathématiques, nouveaux contenus, TIC

\section{Introdução}

As políticas de formação continuada realizadas, de maneira geral, pela administração educativa têm por objetivo que os professores realizem uma prática que seja cada vez melhor, de mais qualidade. Embora existam diferentes políticas de formação continuada, em especial, há dois modelos organizativos claramente diferenciados. No primeiro, realizam-se assessorias no próprio centro educativo, para que se consiga uma reflexão crítica sobre a própria prática, na qual, podem-se derivar mudanças. No segundo caso, com o objetivo de alcançar o desenvolvimento profissional dos docentes, se oferecem cursos de formação permanente, nos quais o professor se inscreve a título pessoal. Neste último aspecto, supõe-se que o desenvolvimento alcançado produzirá uma mudança nas práticas do professor assistente e que, desde sua vez, essas mudanças possam ser levadas a seus colegas de escola/instituição. 
Com relação ao segundo modelo, Moreira (2004) levanta a discussão de que os mestrados acadêmicos atuais não atendem às necessidades emergentes da prática docente e, nesse sentido, defende a ideia da criação dos mestrados profissionais em ensino, visto que esse tipo de mestrado está voltado para professores em exercício, e apresenta um currículo que contempla as áreas específicas do conhecimento e a formação didático-pedagógica.

$\mathrm{Na}$ tentativa de capacitar professores de matemática em exercício em Brasil, seguindo as sugestões do parágrafo anterior, e de atender a meta dezesseis disposta na lei 13.005/2014 referente ao Plano Nacional de Educação (PNE) - de formar cinquenta por cento dos professores da educação básica em nível de pós-graduação até o ano de 2020, (Ministério da Educação, 2014) - iniciou-se, em 2010, por meio da recomendação do Conselho Técnico-Científico da Educação Superior da Coordenação de Aperfeiçoamento de Pessoal de Nível Superior (Capes), o Mestrado Profissional em Matemática em Rede Nacional (PROFMAT).

Este mestrado se constitui como um curso de pós-graduação stricto sensu, semipresencial, oferecido em todo território nacional brasileiro, coordenado pela Sociedade Brasileira de Matemática (SBM) e tem, como principal objetivo, estimular a melhoria do ensino de Matemática em todos os níveis, (Coordenação de Aperfeiçoamento de Pessoal de Nível Superior; 2013; Sociedade Brasileira de Matemática, 2013);

No intuito de contribuir para a melhoria do ensino de matemática, os professores que realizam esse mestrado devem materializar os conteúdos adquiridos no formato de um trabalho de conclusão de curso (TCC), visto que tal TCC busca a inter-relação entre a teoria e a prática. Por esta razão, as orientações fornecidas pelo PROFMAT demonstram que ele deve ser desenvolvido de forma inovadora, de acordo com temas específicos do currículo de Matemática do Ensino Básico e que tenha, preferencialmente, aplicação direta em sala de aula, contribuindo, assim, para o enriquecimento do ensino da disciplina. (Sociedade Brasileira de Matemática, 2013).

No sentido de estudar como se dá a materialização da inter-relação entre o conhecimento teórico e prático dos professores que cursam o PROFMAT, este trabalho tem como objetivo geral determinar quais são, e como se justificam, as inovações nos trabalhos de conclusão de curso do Mestrado Profissional em Matemática em Rede Nacional (PROFMAT), em particular, no contexto do estado do Rio Grande do Sul, Brasil.

A estrutura deste artigo é a seguinte: após esta introdução, em que se explica o objetivo desta investigação, na segunda seção, enumeram-se as tendências que orientam as inovações, define-se a ideia processos de instrução, explica-se a metodologia qualitativa utilizada e, também, apresenta-se uma breve revisão de literatura. Na seção três, realiza-se a análise dos 29 trabalhos de conclusão de curso do estado do Rio Grande do Sul em duas partes: a) na primeira, analisam-se e relacionamse o tipo de inovação proposto (incorporação de novos conteúdos, recursos informáticos, etc.) e o processo de instrução contemplado (planejamento, implementação, redesenho); b) na segunda parte, analisam-se, com detalhe, os argumentos dados pelos autores, para justificar cada um dos tipos de inovação. $\mathrm{O}$ artigo termina com uma seção de considerações finais.

\section{Marco conceitual, metodológico e revisão de literatura}


Nesta seção enumeram-se as tendências que orientam as inovações, define-se a ideia de processos de instrução e, também, apresenta-se uma breve revisão de literatura cujas investigações abordadas tenham como foco as análises de trabalhos finais de mestrados profissionais.

\subsection{Tendências que orientam as inovações no ensino de matemática}

O objetivo final dos programas de formação e desenvolvimento profissional dos professores de matemática é conseguir um impacto na melhoria do ensino de matemática. Aparece, então, o problema do que se deve entender por melhoria do ensino de matemática. Uma maneira implícita de responder esta questão é considerar que a melhora consiste em seguir algumas determinadas tendências atuais sobre o ensino de matemática, já que se considera que o ensino realizado segundo estas tendências é de qualidade. Algumas destas correntes são específicas do ensino de matemática, enquanto outras são aplicáveis, inclusive, em outras áreas do conhecimento.

Recentemente, na área da Educação Matemática, aumentou-se o interesse sobre quais são as tendências em que os professores devem seguir em suas aulas para que consigam um ensino de qualidade. Tais tendências podem ser inferidas das publicações mais relevantes da área - por exemplo, handbooks sobre investigação em Educação Matemática (Bishop, Clements, Keitel, Kilpatrick, \&Leung, 2003; English, BartoliniBusi, Jones, Lesh, \& Tirosh, 2008; Gutiérrez \& Boero, 2006; Lester, 2007), ou publicações da série ICMI studies (Batanero, Burrill, \& Reading, 2011; Fauvel \& Maanen, 2000; Hanna, 1996; Holton, 2001; Mammana \& Villani, 1998; Stacey, Chick, \& Kendal, 2004), a criação de Topic Study Group, em congressos (por exemplo, o TSG4 New developments and trends in mathematics education at upper secondary level do ICME 11), ou na publicação de revistas relacionadas, especificamente, com essa temática (como o Journal of Mathematics Education Trends and Research). Por outra parte, diversos autores do campo da Educação Matemática têm refletido de maneira sistemática sobre quais são as novas perspectivas atuais no ensino de matemática (Font, 2008; Guzmán, 2007; Müller, 2000). Estes autores, embora apresentem algumas diferenças nas tendências que assumem como relevantes, coincidem em na maioria delas.

Estas novas correntes foram cristalizadas nos currículos de distintos países sob a forma de orientações e princípios que nos dizem, a priori, como se deve ter um ensino de matemática de qualidade. O caso paradigmático de reconversão de algumas destas tendências em princípios, pode ser encontrado nos princípios e padrões do National Council of Teachers of Mathematics (NCTM, 2000). Além disso, elas tiveram seu impacto nas orientações oficiais em diferentes estados de Brasil - por exemplo, as diretrizes curriculares da Secretaria de Estado da educação do estado do Paraná (2008) apresentam as tendências metodológicas que compõem o campo de estudo da Educação Matemática: etnomatemática, modelagem matemática, mídias tecnológicas, história da matemática, investigação matemática e resolução de problemas - e sua influência é clara nos Parâmetros Curriculares Nacionais (Ministério da Educação, 2000).

Nesta investigação partimos do pressuposto que há uma pressão para que estas novas perspectivas sejam assumidas pelos professores que estão em exercício e, também, para que sejam levadas em conta na formação de futuros professores, já que 
se considera que o ensino realizado, segundo as mesmas, é de qualidade. Por isso, quando os professores têm que propor e justificar uma inovação didática, recorrem, de forma implícita ou explícita, a elas, as quais se fazem presentes nas orientações curriculares, cursos de formação, etc.

As tendências principais que tivemos em conta neste estudo são as seguintes: a incorporação de novos conteúdos; apresentação de uma matemática contextualizada; dar importância ao ensino de processos matemáticos (resolução de problemas, modelagem matemática); ensino e aprendizagem do tipo ativo (construtivista); considerar que saber matemática implica ser competente em aplicá-la a contextos extramatemáticos; princípio da equidade na Educação Matemática obrigatória e incorporação das novas tecnologias da informação e da comunicação (TIC). $\mathrm{Na}$ continuação comentamos brevemente as duas que orientam a maioria dos Trabalhos de Conclusão de Curso (TCC): incorporação de novos conteúdos e as TIC.

Ultimamente se observa, na Educação Básica, um aumento de conteúdos de Geometria, Estatística, Probabilidade e Matemática Discreta (Font, 2008; Guzmán, 2007) - um exemplo paradigmático é o novo currículo de bacharelado do Equador, o qual contempla quatro blocos curriculares: números e funções; álgebra e geometria; matemática discreta; probabilidade e estatística.

A necessidade de uma recuperação dos conteúdos geométricos no ensino de matemática é algo em que todos os interessados no ensino de matemática parecem coincidir. Também se observa um encaminhamento no aumento ou ampliação dos conteúdos de estatística e probabilidade. Esta é uma dinâmica na qual todos os sistemas educativos parecem concordar e, efetivamente, são muitos os países que incluem estes conteúdos em seus programas de Ensino Médio. Também se considera que determinadas partes da Matemática Discreta são suficientemente elementares para poder formar parte, com êxito, do ensino não universitário. A combinação clássica, assim como os aspectos modernos dela, tais como a teoria de grafos ou a geometria combinatória, juntamente com a teoria elementar dos números, são consideradas as mais adequadas para serem implementadas no ensino de matemática da Educação Básica.

Outra tendência que se observa, ao nível geral, é a incorporação das TIC no ensino de matemática (Font, 2008; Guzmán, 2007) e também na formação de professores (Llinares, 2012). Esta incorporação afeta tanto os novos conteúdos matemáticos, quanto àqueles que sempre formaram parte do currículo. A introdução de novas formas de fazer matemática, geradas pelo uso das TIC, pode levar os alunos a desenvolverem novas maneiras de pensar e de resolver determinadas tarefas, visto que podem passar a trabalhar, em maior escala, com representações gráficas, testar hipóteses, etc. Embora, em um primeiro momento, pensava-se, de maneira ingênua, que tudo se transformava em vantagens quando da incorporação das TIC, ao passo que em um segundo momento, superou-se esta ingenuidade, pois se por um lado as novas tecnologias no ensino de matemática podem facilitar o desenvolvimento de uma nova atitude matemática, podem também gerar obstáculos epistemológicos - principalmente no que tange à questão dos erros e das ambiguidades - ao entendimento de determinados conceitos (Font, 1999; 2008).

\subsection{Sobre os processos de instrução}


A melhoria do ensino de matemática se concretiza em um processo de instrução, o qual implica, entre outros aspectos, no planejamento, implementação e redesenho de uma sequência de tarefas para serem trabalhadas em uma determinada aula.

A primeira fase de um processo de instrução é o desenho de uma sequência de tarefas para ensinar um determinado tema. Esta sequência deve ter em conta: um tempo determinado, um público a quem se destina e o detalhamento das atividades propostas. A segunda fase é a implementação deste planejamento. Já, a terceira fase é a avaliação do processo de instrução realizado e, se for o caso, o seu redesenho.

Segundo Godino, Contreras e Font (2006), tanto o planejamento como a implementação de um processo de instrução sobre um conteúdo ou tema matemático implica em uma sequência de configurações didáticas. Estas configurações estão associadas a três aspectos: (I) configuração epistêmica, que refere-se a uma tarefa, aos procedimentos para sua resolução, linguagens, conceitos, proposições, argumentações; (II) configuração instrucional, que diz respeito à rede de objetos docentes, discentes e mediacionais postos em jogo, quando da tarefa matemática abordada; (III) configuração cognitiva, rede de objetos intervenientes e emergentes dos sistemas de práticas pessoais que se colocam em jogo na implementação de uma configuração epistêmica. Por outra parte, Godino, Font, Wilhelmi e Castro (2009), argumentam que uma fase importante dos processos de instrução é sua avaliação e redesenho, mediante critérios de idoneidade, que permita incidir na melhora das futuras implementações.

\subsection{Alguns estudos focados na análise de trabalhos finais de curso da área de educação em ciências e matemática}

$\mathrm{Na}$ maioria dos países, os mestrados profissionais em ensino que incluem um trabalho final de curso, são relativamente recentes. Por essa razão, não há um número expressivo de pesquisas baseadas nas análises desse tipo de produção final. Investigações brasileiras como as de Cevallos (2011), Ferreira (2013), Schäfer (2013) e Prado, Silva e Araújo (2011) tratam de analisar os impactos que programas de pósgraduação em Ciências e Matemática ocasionam na formação continuada de professores. A título de exemplo, em Prado, Silva e Araújo (2011) descrevem-se e analisam-se impactos causados pela formação de mestres em Ensino de Ciências Naturais e Matemática que atuam no Instituto Federal de Educação, Ciência e Tecnologia do Rio Grande do Norte, formados no mestrado profissional da Universidade Federal do Rio Grande do Norte (UFRN). Por meio da análise dos trabalhos finais, puderam especificar as áreas, focos e subfocos temáticos abordados pelos professores, como tais professores se projetam no seio da Instituição, verificaram as inovações propostas pelos mesmos, as possíveis mudanças de percepção e de atitudes e o investimento em ações formativas para outros professores.

\section{Os critérios de idoneidade didática como organizadores da reflexão nos trabalhos finais de curso da área de Matemática}

No caso específico da área da matemática, na Espanha, também se apresentam investigações voltadas à formação de futuros professores de secundária que se baseiam na análise dos trabalhos finais de mestrado. Na sequência, resumimos as investigações que foram realizadas nas universidades de Granada e de Barcelona. 
Recentemente, na Universidade de Granada, foram realizados alguns trabalhos finais de mestrado (TFM) que avaliam os processos de instrução utilizando os critérios de idoneidade didática propostos pelo Enfoque Ontosemiótico da Cognição e Instrução Matemática (EOS) (Godino, Batanero e Font, 2007; Godino, Contreras e Font, 2006). Para a avaliação de processos de instrução, tal enfoque propõe como ferramenta essencial a noção de idoneidade didática (Breda, Font e Lima, 2015; Godino, 2013; Godino, Batanero e Font, 2007; Godino, Contreras e Font, 2006), a qual permite avaliar um processo de instrução e responder à pregunta: quais mudanças devem ser realizadas no planejamento e implementação do processo de instrução para que se consiga uma melhora nas futuras implementações? A idoneidade didática de um processo de instrução define-se como o grau no qual tal processo (ou uma parte dele) reúne certas características que permitem qualificá-lo como idôneo (ótimo ou adequado) para que se consiga a adaptação entre os significados pessoais adquiridos pelos estudantes (aprendizagem) e os significados institucionais pretendidos ou implementados (ensino), tendo em conta as circunstancias e recursos disponíveis (entorno). A noção de idoneidade didática pode ser decomposta em seis idoneidades específicas:

Epistêmica: refere-se a que a matemática ensinada seja "boa matemática". Para isso, além de tomar como referência o currículo prescrito, trata-se de tomar como referência a matemática institucional que se transposta no currículo; Cognitiva: expressa o grau em que as aprendizagens pretendidas/implementadas estão na zona de desenvolvimento potencial dos alunos, assim como a proximidade das aprendizagens adquiridas às que foram pretendidas ou implementadas; Emocional: distribuição temporal dos estados afetivos (atitudes, emoções, afetos, motivações) de cada aluno em relação com os objetos matemáticos e com o processo de estudo seguido; Interacional: grau em que os modos de interação permitem identificar e resolver conflitos de significado e favorecem a autonomia da aprendizagem; Mediacional: grau de disponibilidade e adequação dos recursos materiais e temporais necessários para o desenvolvimento do processo de ensino-aprendizagem; Ecológica: grau de adaptação do processo de estudo ao projeto educativo do centro, às diretrizes curriculares, às condições do entorno social; (Font, Planas e Godino, 2010, p.101).

Para cada um destes critérios, sugere-se um sistema de indicadores associados que podem ser avaliados em uma escala de um a três, por exemplo. Trata-se de um sistema que permite avaliar ou auto-avaliar de maneira completa e equilibrada os elementos que, em conjunto, compreendem um processo de instrução de qualidade na área da matemática. Por essa razão, são ferramentas úteis para serem usadas nos trabalhos finais de mestrado.

Em Posadas e Godino (2014), descreve-se o processo formativo realizado pela primeira autora, sob a supervisão e o apoio do segundo autor, enfatizando o papel da noção de idoneidade didática como um instrumento de reflexão sistemática sobre a própria prática. Esses autores explicam o foco do TFM realizado por Posadas (2013), pois esta apresentou uma reflexão sobre a sua experiência vivenciada na fase das práticas de ensino, na qual, teve a oportunidade de assumir a responsabilidade sobre trabalhar o tema referente ao estudo da função quadrática no terceiro ano do Ensino Secundário Obrigatório (ESO). A finalidade do estudo foi a de obter critérios para o replanejamento da unidade didática, de tal forma que permitissem introduzir mudanças no ensino do tema acima citado.

Durante os cursos 2009-2010 a 2012-2013, no Máster de Formación de Profesores de Secundaria de Matemáticas de la Universitat de Barcelona, e durante os cursos 2013-2014 a 2015-2016 no Máster Interuniversitario de Formación de Profesores de 
Secundaria de Matemáticas de Catalunya, os formadores de futuros professores sugeriram a seus alunos o uso de uma rubrica baseada nos critérios de idoneidade propostos pelo EOS para:

- Avaliar sua prática;

- Desenhar, no trabalho final de mestrado, uma proposta de melhora da unidade didática, implementada no período de práticas, que melhore alguns aspectos que a avaliação anteriormente realizada indica que devem ser melhorados.

Ferreres e Vanegas (2015) e Giménez, Vanegas, Font e Ferreres (2012) realizaram uma investigação com futuros professores de secundária em seus trabalhos finais de mestrado (TFM) sobre como eles usavam critérios de idoneidade. Nessa investigação observou-se que os critérios de idoneidade foram uma ferramenta útil para organizar a reflexão dos futuros professores sobre sua própria prática. Em outras palavras, os alunos não foram conscientes da potência dos critérios como ferramentas a priori para desenhar uma sequência didática. No entanto, os critérios serviram para que fizessem a reflexão sobre sua prática realizada anteriormente, se fazendo úteis quando os professores tiveram que justificar uma sequência didática que melhoraria a implementação realizada no seu período de prática. A elaboração de indicadores de idoneidade para ensino da matemática está provando ser uma ferramenta relevante na formação de professores de matemática, como é mostrado em Breda, Font e Lima (2015).

Os trabalhos de investigação apresentados acima, tanto no âmbito da formação de professores de ciências, quanto no âmbito da formação de professores de matemática, sugerem que a análise das produções finais de curso permite verificar, dentre outros aspectos, a inovação das práticas, as mudanças de percepção e de atitudes dos professores que realizaram o mestrado, a avaliação que os professores fazem de suas propostas didáticas e as justificativas, dadas por eles, a fim de possibilitar a melhoria do ensino de matemática. Nesta linha, a pesquisa que aqui se apresenta, trata de investigar a forma que os professores conceituam e justificam a inovação em seus trabalhos de conclusão de curso.

\subsection{Aspectos metodológicos}

Para alcançar o objetivo proposto, este trabalho apóia-se em uma metodologia de investigação qualitativa, conforme Ludke e André (1986), que se baseia na compreensão e interpretação dos dados. Foram considerados dois aspectos qualitativos, o primeiro é o tipo de inovação, na qual se considerou as sete de tendências explicadas anteriormente. $O$ segundo aspecto é o que chamamos de processo de instrução contemplado (planejamento, implementação e redesenho).

Para selecionar os trabalhos analisados, primeiramente, observou-se a distribuição de universidades participantes do programa com o seguinte resultado: em 13 estados há a participação de apenas uma universidade, em quatro estados há a participação de duas universidades e nos demais estados há a participação de três ou mais universidades. Em um segundo momento, selecionou-se o estado do Rio Grande do Sul, pois, por um lado, este participa do programa por meio de duas universidades (Universidade Federal de Santa Maria e Universidade Federal de Rio Grande), e por outro apresenta um número de produções razoável para que se possa inferir conclusões a respeito (29 TCC); além disso, há facilidade de acesso aos documentos eleitos para a análise. Por tanto, selecionaram-se 29 TCC realizados e publicados no estado do Rio 
Grande do Sul, do primeiro semestre de 2013 ao segundo semestre de 2014, de acordo com as seguintes fases descritas.

Tal como sugerimos na seção 2.3 , essa investigação partiu da suposição de que o TCC era um espaço claramente avaliativo, já que os professores deveriam apresentar uma proposta de melhora e deveriam realizar uma análise em didática para justificar sua qualidade. Considerou-se que, quando as opiniões atribuem certo juízo de valor, há evidencias do uso de alguns indicadores presentes nos componentes de idoneidade didática propostos pelo EOS (idoneidade epistêmica, mediacional, ecológica, emocional, interacional e cognitiva); e, além disso, há evidências de que a avaliação positiva destes indicadores está baseada na suposição implícita ou explicita de que existem determinadas tendências relacionadas ao ensino de matemática que nos indicam como deve ser um ensino de matemática de qualidade (Font, 2008; Guzmán, 2007).

Para que os critérios de idoneidade se tornem operativos é necessário definir um conjunto de indicadores observáveis os quais permitam avaliar o grau de idoneidade de cada uma das facetas dos processos de estudo. Por exemplo, todos nós concordamos que é importante implementar uma "boa matemática". Contudo, podemos entender de distintas maneiras o que significa uma "boa matemática". Para alguns critérios, os descritores são relativamente fáceis de consensuar (o critério de meios, por exemplo), para outros, como o caso da idoneidade epistêmica, é mais difícil. Godino (2013) y Godino, Bencomo, Font y Wilhelmi (2007) apresentam um sistema de indicadores que serve de guia de análise e avaliação da idoneidade didática. No Máster em Formación de Profesores de Secundária en Matemáticas da Universidade de Barcelona, de 2010 a 2014, e no Máster Interuniversitario en Formación de Profesores de Secundaria en Matemáticas da Catalunha de 2014 a 2016, utilizou-se uma reformulação (Font, 2015) da pauta proposta em Godino, Bencomo, Font e Wilhelmi (2007), que é a que utilizaremos nesta pesquisa e que também foi utilizada nas pesquisas de Seckel (2016), Breda, Pino-Fan e Font (2016) e Breda (2016). Na continuidade apresentamos, na tabela 1 (Font, 2015, p. 1), como exemplo, os componentes do critério de idoneidade epistémico e seus respectivos descritores.

Tabela 1. Componentes e descritores da idoneidade epistêmica (Font, 2015, p. 1)

\begin{tabular}{ll}
\hline \multicolumn{1}{c}{ Componentes } & \multicolumn{1}{c}{ Descritores } \\
\hline Erros & $\begin{array}{l}\text { Não se observam práticas que são consideradas incorretas do ponto de vista } \\
\text { matemático. }\end{array}$ \\
Ambiguidades & $\begin{array}{l}\text { Não se observam ambiguidades que possam levar os alunos a cometerem } \\
\text { confusões, conforme segue: definições e procedimentos claros e e } \\
\text { corretamente enunciados, adaptados ao nível educativo ao qual se dirigem; } \\
\text { adequação das explicações, comprovações, demonstrações no nível } \\
\text { educativo ao qual se dirigem; uso controlado de metáforas, etc. }\end{array}$ \\
Riqueza de processos & $\begin{array}{l}\text { A sequência de tarefas contempla a realização de processos relevantes para a } \\
\text { atividade matemática (modelação, argumentação, resolução de problemas, } \\
\text { conexões, etc.). }\end{array}$
\end{tabular}


Representatividade

Os significados parciais (definições, propriedades, procedimentos, etc.) são uma amostra representativa da complexidade da noção matemática que se quer ensinar contemplada no currículo;

Os significados parciais (definições, propriedades, procedimentos, etc.) são uma amostra representativa da complexidade da noção matemática que se quer ensinar;

Para um ou vários significados parciais há uma amostra representativa de problemas;

Para um ou vários significados parciais, há o uso de diferentes modos de expressão (verbal, gráfico, simbólico, etc.), tratamentos e conversões entre eles.

Em uma primeira fase de esta investigação, para cada um dos 29 TCC foram analisadas as razões que o autor utiliza para justificar sua proposta inovadora. Em Breda e Lima (2016), pode-se consultar um exemplo de dita análise, a qual, basicamente, consiste em selecionar evidências nas quais o autor trata de justificar que a sequência de tarefas propostas em seu TCC representa uma melhora no ensino do objeto matemático considerado em sua proposta - que podem ser consideradas evidências de uso implícito de alguns dos descritores dos diferentes critérios de idoneidade didática propostos pelo EOS.

$\mathrm{Na}$ continuação apresentamos um exemplo de como foram consideras certas evidências relacionadas aos componentes e descritores da idoneidade didática em determinados parágrafos de um TCC analisado no presente trabalho.

O trabalho intitulado Um relato sobre à introdução às somas de Riemann na Educação Básica (Lopes, 2014) apresenta o desenho e a implementação de uma proposta didática para uma turma de alunos do terceiro ano do Ensino Médio para introduzir, intuitivamente, o cálculo integral por meio do estudo das áreas de figuras geométricas planas com contorno curvilíneo. Trata-se de ampliar o cálculo de áreas que se estuda no Ensino Médio com o cálculo de áreas de figuras de contorno curvilíneo mediante o uso do método de Arquimedes e o método de Riemann.

$\mathrm{O}$ autor escreve alguns enunciados que, em nossa análise, foram considerados como evidencias relacionadas ao componente riqueza de processos (idoneidade epistêmica), já que justifica que a qualidade de sua proposta é inovadora, pois fomenta que os alunos realizem processos matemáticos relevantes, em particular, o processo de modelagem matemática, (Lopes, 2014, p. 22):

[...] Busca-se, com isso, construir com o aluno, por meio do uso de modelos matemáticos, conceitos impostos na maioria das vezes. Além disso, pretende-se direcionar tais modelos de tal forma que permitam introduzir conceitos de matemática avançada, no caso específico, a introdução do cálculo integral, por meio do cálculo de área.

Para conseguir o objetivo geral deste trabalho, explicitado na introdução, na segunda parte da análise, descrita à continuação, analisaram-se e relacionaram-se, por uma parte, o tipo de inovação proposto (incorporação de novos conteúdos, recursos 
informáticos, etc.) e, por outra parte, o processo de instrução contemplado (desenho, implementação e redesenho).

\section{Análise dos trabalhos de conclusão de curso}

Sobre os processos de instrução, dos 29 trabalhos analisados, 13 apresentam apenas o planejamento, 11 apresentam planejamento e implementação da proposta, um apresenta planejamento, aplicação e redesenho e quatro não apresentam processo de instrução.

Com relação ao tipo de inovação, dos TCC analisados, 13 baseiam a sua proposta de inovação para o Ensino Básico na incorporação de conteúdos matemáticos de nível superior; três propõem a realização de conexões intramatemáticas; seis dos TCC baseiam na conexão da matemática com contextos extramatemáticos; cinco consideram, explicitamente, o uso das TIC como núcleo de sua proposta inovadora; um deles na introdução de materiais visuais e manipulativos e um TCC baseia a sua inovação no princípio da equidade.

$\mathrm{Na}$ continuação nos centraremos nos grupos majoritários que são o da inovação matemática (incorporação de conteúdos de nível superior) e o da incorporação de recursos tecnológicos (TIC) a fim de analisar o tipo de justificativa que os professores dão sobre a qualidade de suas inovações. Para uma análise detalhada dos outros tipos de inovação, pode-se consultar Breda (2016).

\subsection{Da incorporação de conteúdos de matemática de nível superior na Educação Básica}

A análise realizada permite concluir que dos 29 trabalhos, quase a metade deles (13) baseiam sua proposta inovadora na incorporação de conteúdos de nível superior na Educação Básica, especialmente, conteúdos relacionados à Matemática Discreta e Introdução ao Cálculo. Nove dos 13 trabalhos contemplam processos de instrução, dos quais dois explicam apenas o planejamento e sete explicam o planejamento e implementação.

Dos 13 trabalhos que propõem como inovação a incorporação de conteúdos matemáticos de nível superior na Educação Básica, quatro não apresentam processo de instrução, pois focalizam suas propostas em um trabalho de cunho matemático. Dois deles entendem que incorporar conteúdos de ensino superior na Educação Básica especialmente conteúdos relacionados à Matemática Discreta e com a recuperação de teoremas e procedimentos sugeridos pela história da matemática - melhora o ensino de matemática, pois amplia os conhecimentos dos alunos, auxilia na construção de conceitos e entusiasma os estudantes. Embora as justificativas dadas estejam centralizadas no critério emocional, é importante salientar que os trabalhos que não apresentam a fase de implementação mostram certa superficialidade no uso dos critérios epistêmico e ecológico. Além disso, é notável a falta de reflexão em relação aos critérios interacional e cognitivo, por exemplo:

Mas também fortaleceu a convicção de que se pode e deve trabalhar com "temas novos", que estão ou não diretamente relacionados com os conteúdos programáticos. Pois estes têm a capacidade de entusiasmar estudantes, despertá-los, ampliar seus conhecimentos. (Pinz, 2013, p.44). 
Como as ideias de Arquimedes são intuitivas, podemos inferir que podem ser usadas por estudantes que não viram cálculo integral na escola média, para construir os conceitos de áreas mais gerais. (Mohnsam, 2014, p. 73).

A primeira evidencia acima nos mostra a justificativa dada por meio da idoneidade emocional e em menor medida, com a ecológica. Na segunda evidência podemos ver com maior clareza o componente de idoneidade ecológica, pois o autor tenta estabelecer uma conexão entre o cálculo de Arquimedes com o currículo de educação básica, visto que tal conteúdo apresenta uma abordagem intuitiva que pode ser trabalhada na Educação Básica. É importante salientar que os critérios contemplados nas propostas que não foram implementadas, se relacionam, sobre tudo, com o critério de idoneidade epistêmica, ecológica e, em menor medida, com o critério mediacional e que outros critérios como o cognitivo, o emocional e o interacional aparecem muito pouco desenvolvidos.

Já, os sete trabalhos que foram implementados consideram a melhoria do ensino de matemática através da incorporação de conteúdos matemáticos do ensino superior na Educação Básica - relacionados com recorrência, indução matemática, introdução ao cálculo diferencial e integral, geometria projetiva, equaciones cúbicas e método de mínimos quadrados, pois estes permitem, segundo os autores: a) estabelecer processos relevantes como a modelação de situações extramatemáticas, ou seja, modelar matematicamente situações do cotidiano; b) a construção de conceitos e estabelecimento de relações intramatemáticas, ou seja, relacionar o conteúdo que está sendo ensinado (de nível superior) com conteúdos matemáticos habitualmente trabalhados na Educação Básica; e c) a ampliação do olhar do estudante, de modo que a introdução de ideias intuitivas relacionadas a conteúdos do ensino superior facilite seus estudos posteriores (na universidade). Pelo fato de os trabalhos terem sido implementados, observa-se o uso dos critérios de forma mais equilibrada. Por um lado, os autores realizam uma reflexão epistêmica e ecológica mais aprofundada, sem deixar de lado outros critérios que devem apresentar relevância quando da implementação de uma sequência didática, como o cognitivo, interacional, emocional e mediacional.

[...] neste trabalho, são propostas várias atividades que trazem as equações de recorrências para a sala de aula - possibilitando ao aluno ter acesso a problemas do cotidiano - as quais serão resolvidas pelos alunos com o auxílio de planilhas eletrônicas deduzindo o próximo valor de cada célula, ou seja, o próximo valor da sequência, sempre em função do termo anterior ou de dois termos anteriores. (Martins, 2014, p. 14).

[...] No segundo momento, após, um breve debate sobre as questões que foram levantadas pelos estudantes acerca da duplicação, foi solicitado aos alunos que representassem, através de um desenho, uma auto estrada, tendo como referência o trecho em duplicação da BR 392. A atitude esperada era que os estudantes representassem a auto estrada como segmentos de retas paralelos ou linhas que não possuam pontos em comum[...] (Gonçalves, 2013, p. 102-104).

Acredita-se que, a proposta se mostra muito eficiente e inteligente. Pois, aos utilizar códigos, os alunos se sentem desafiados como num jogo, e sabe-se que tal envolvimento se mostra difícil de acontecer nas aulas tradicionais de matemática. (Souza, 2014, p. 24).

No decorrer dos encontros com os estudantes, foi possível relacionar vários aspetos de real aplicação com os problemas e atividades que eram realizados, e percebeu-se que isto fez sentido para os estudantes, na medida em que eles conseguiam fazer as relações e concluir sobre o que se pedia. (Molon, 2013, p. 112). 
Em função das dificuldades resultantes da abordagem destes conteúdos um tanto abstratos, fez-se importante a elaboração de uma prática pedagógica adequada, capaz de tornar o aluno apto a compreender todos os conceitos e propriedades envolvidas no processo. A partir deste momento, passaremos a destacar todas as atividades desenvolvidas, bem como as dúvidas elencadas pelos discentes em todo o processo. (Souza, 2013, p. 23).

Desta forma, no trabalho de aplicação, dividido em três etapas, busca-se construir o conhecimento mediante o uso de modelos matemáticos. A partir da primeira construção, à medida que o assunto é aprofundado e que novos elementos surgem, outros modelos são construídos, embasados nos anteriores. [...] (Lopes, 2014, p.22).

As evidências acima demonstram, de forma geral que, as propostas implementadas contemplam, em maior medida, o uso de todos os critérios de idoneidade propostos pelo Enfoque Ontosemiótico. Martins (2014), por exemplo, apresenta uma reflexão que vai em direção ao uso das planilhas eletrônicas com a finalidade de resolver problemas, explorando o uso do critério mediacional. Já, Gonçalves (2013), organiza sua proposta de tal forma que haja diálogo entre professor e alunos, enfatizando, assim, o critério interacional. Souza (2014) destaca o aspecto emocional, Souza (2013) o cognitivo, Molon (2013) o ecológico e Lopes (2014) o epistêmico. Nesse sentido, é possível salientar que os professores que implementaram a proposta manejam maior quantidade de critérios e seus respectivos descritores, além de aprofundarem-se de maneira significativa em alguns componentes relacionados a determinado critério.

\subsection{Da incorporação das TIC}

Com relação à quantidade de trabalhos que incorporam as TIC em suas propostas de inovação didáticas, encontramos que, dos 29 trabalhos publicados, 20 apresentam o uso de tais tecnologias, ou seja, aproximadamente dois terços de todas as produções realizadas em dito estado.

Quanto ao tipo de recursos que são contemplados em tais propostas, observamos que os recursos das TIC mais utilizados são programas dinâmicos gratuitos para trabalhar geometria, em particular, onze trabalhos incorporam o software GeoGebra e um incorpora o Winplot e o wxMáxima; e a planilha eletrônica (cinco trabalhos) para trabalhar conteúdos de aritmética e funções; um trabalho aborda o uso dos widgets em um questionário para o estudo de probabilidade. Este resultado coincide com revisões realizadas sobre o uso das TIC em outros países. Font (2011), por exemplo, realizou um estudo sobre as investigações realizadas na Espanha em relação à introdução das TIC no Ensino Secundário Obrigatório (ESO) e a conclusão que chega é a de que as investigações usam, sobretudo, esses tipos de programas informáticos.

Embora, de alguma maneira, 20 destes trabalhos incorporem as TIC em suas propostas, apenas cinco deles consideram, explicitamente, o uso das TIC como o núcleo de sua proposta inovadora. Estes cinco trabalhos propõem diferentes fases do processo de instrução. A maioria (quatro deles) apenas informa a incorporação das TIC na fase do planejamento, visto que estas propostas não foram implementadas em sala de aula. Nenhum apresenta o planejamento e a aplicação e apenas um apresenta o planejamento, implementação e redesenho da sequência didática e, neste caso, o autor reflete sobre o uso das TIC na fase do redesenho de sua proposta.

Os quatro que propõem apenas o planejamento entendem que o uso das TIC melhora o ensino de matemática, pois tornam as propostas atrativas que motivam os 
alunos, promovem um aprendizado construtivista e significativo, possibilitam a interação entre professor-aluno-software e contribuem para a apropriação do saber matemático dos alunos, visto que auxiliam na resolução de problemas.

Nossa proposta visa usar esse formato para desenvolver um questionário que ao mesmo tempo prenda a atenção do estudante e estimule a construção do conhecimento em probabilidade (Jung, 2013, p.15).

A utilização do software GeoGebra e de outras tecnologias no estudo da trigonometria no Ensino Fundamental pode ser um excelente recurso para auxiliar os professores nas suas aulas, tornando-as mais atrativas e significativas aos olhos dos alunos (Strasburg, 2014, p.122).

Nos excertos acima é possível notar que, na reflexão dos professores que apresentam somente o planejamento da proposta, salientam-se as justificativas baseadas, sobretudo, no critério emocional e, de maneira superficial, nos critérios cognitivo, interacional e epistêmico. $\mathrm{O}$ aspecto ecológico, por exemplo, aparece muito pouco desenvolvido. Já, o trabalho que apresenta o planejamento, implementação e redesenho, considera a melhoria do ensino de matemática através do uso do Software GeoGebra, principalmente pela riqueza de processos que este instrumento oferece, pois, segundo a autora, este permite apresentar uma geometria dinâmica; permite conectar Geometria com Álgebra; promove a aprendizagem autônoma e construtivista e permite a realização de investigação matemática, servindo como uma ferramenta para verificar resultados.

[...] o uso de tecnologia digital, especificamente softwares de geometria dinâmica levam o aluno a pensar e a vincular o que ele já sabe com o novo conhecimento a adquirir [...] esse tipo de intervenção pode ser chamado de investigação matemática, pois o objetivo é descobrir relações entre objetos matemáticos conhecidos e desconhecidos, procurando identificar propriedades. (Bastos, 2013, p.17).

[...] acreditamos que o melhor software é o GeoGebra, para trabalhar com Geometria Analítica, pois além de ser gratuito e ter atualizações periódicas, o modo como ele relaciona geometria e álgebra é apropriado ao estudo da Geometria Analítica.[...] Também possui a vantagem da geometria dinâmica, que permite mover objetos e aplicar diversas transformações e automaticamente enxergar a mudança nas equações, assim como com áreas, ângulos, rotações, translações, etc. (Bastos, 2013, p. 28).

Devido ao tempo e ainda a necessidade de introduzir o GeoGebra teríamos que excluir algo da lista. Optamos por inserir todos os conteúdos nos três dias de atividades e distribuir o que é essencial no início de cada atividade, prevendo o estudante que não tivesse tempo de fazer tudo, ao menos teria feito a parte essencial. (Bastos, 2013, p. 93).

Bastos (2014) contempla em sua proposta, especialmente na fase do redesenho, todos os critérios de idoneidade de forma equilibrada. Aprofunda-se de maneira significativa em alguns componentes relacionados a determinado critério e preocupase com o equilíbrio no uso dos diferentes critérios (epistêmico, cognitivo, mediacional, interacional, emocional e ecológico), incorporando, inclusive, os critérios que não foram contemplados durante a implementação, no redesenho, como no caso do ajuste do tempo de realização das atividades. 
A partir dos excertos expostos acima percebemos que, no discurso dos professores, aparece, de maneira geral e um pouco ingênua que o uso das TIC, em suas propostas didáticas, promove a melhoria do ensino de matemática, pois estes recursos estimulam, motivam, tornam as aulas atrativas e são ferramentas que facilitam a abordagem de conceitos e a resolução de problemas. Contudo, há poucas evidências que indicam que a incorporação de tais recursos promove uma riqueza de processos matemáticos relevantes e que esse tipo de ferramenta auxilia o aluno nas atividades relacionadas ao mundo laboral.

Além dos trabalhos apresentarem certa reflexão em torno dos critérios de idoneidade propostos pelo EOS, é importante destacar que, tanto a incorporação de conteúdos de Ensino Superior na Educação Básica quanto à incorporação das TIC como propostas de inovação corroboram com algumas das tendências que nos indicam como deve ser um ensino de matemática de qualidade, como a tendência de incorporar recursos informáticos e implementar novos conteúdos matemáticos na educação básica (Font, 2008; Guzmán, 2007).

\section{Conclusões}

Este trabalho teve como objetivo determinar quais são, e como se justificam, as inovações nos trabalhos de conclusão de curso do Mestrado Profissional em Matemática em Rede Nacional (PROFMAT). Trata-se de uma investigação que vai ao encontro de outros trabalhos que investigaram a reflexão de professores por meio da análise dos trabalhos finais de mestrado na formação de professores na área de Ciências e Matemática (Cevallos, 2011; Ferreira, 2013; Prado, Silva, \& Araújo, 2011).

Ao analisarmos as produções publicadas pelas universidades participantes do PROFMAT, concluímos que, embora apareçam outras inovações - incorporação de materiais manipulativos e introdução à cidadania e pensamento crítico - a maioria dos trabalhos assume a ideia de inovação matemática (a incorporação de conteúdos de nível superior, conexões intramatemáticas e conexões extramatemáticas) e na inovação a partir do uso de recursos informáticos. Resulta significativo a ausência de outras maneiras de entender a inovação no ensino de matemática. Por exemplo, apenas um trabalho assume sua proposta inovadora no princípio da equidade e nenhum dos trabalhos aborda a ideia de inovação no âmbito de novas estratégias avaliativas ou na organização e gestão das classes.

Com relação ao tipo de inovação majoritário (incorporação de conteúdos matemáticos de nível superior na Educação Básica) os autores dos trabalhos entendem a inovação do ensino de matemática em termos de uma mudança do conteúdo matemático, na qual permite que se ensine uma "matemática de maior qualidade", onde a qualidade, na maioria dos casos, está interpretada em termos da riqueza de processos, compreendido - "[...] no sentido de que a resolução das tarefas propostas implica à realização de processos relevantes na atividade matemática, como a argumentação, a resolução de problemas, a modelação e, em particular, o processo de conexão" (Rondero e Font, 2015, p. 45, tradução nossa).

Quanto às TIC, observa-se que a maioria dos trabalhos incorpora, basicamente, dois tipos de recursos informáticos: GeoGebra e planilha eletrônica. Resulta significativa a pouca presença das possibilidades gratuitas oferecidas pela internet como o uso dos widgets (apenas um trabalho), applets, e-books, plataformas de ensino à distância (como o moodle), fóruns, chats, redes sociais, blogs, busca de informações 
através da internet (um trabalho), etc. Observamos, também, a nula presença dos pacotes informáticos de cálculo simbólico, como por exemplo, MATLAB, MAPLE ou DERIVE. Embora em alguns casos, a justificativa dada pelos professores, para argumentar que a incorporação das TIC representa uma melhora no ensino de matemática, esteja baseada que seu uso permite uma matemática mais rica em termos de processos, em na maioria dos trabalhos se observa que tal justificativa está voltada ao efeito motivador que as TIC produzem nos alunos.

Por fim, gostaríamos de ressaltar que o aspecto relacionado aos processos de instrução apresenta um papel secundário quando da realização dos TCC, pois dos 29 trabalhos, quatro deles não apresentem nenhum processo de instrução, 13 se limitem apenas ao planejamento, somente 11 realizem a implementação da proposta e apenas um, apresente uma reflexão sobre seu redesenho. É importante salientar que os critérios contemplados nas propostas que não foram implementadas, se relacionam, sobre tudo, com o critério de idoneidade epistêmica, ecológica e, em menor medida, com o critério mediacional e que outros critérios como o cognitivo, o emocional e o interacional aparecem muito pouco desenvolvidos, visto que as reflexões relacionadas a estes últimos, só aparecem de maneira mais elaborada na reflexão dos autores que realizam a implementação e/o redesenho da sequência didática.

\section{Referências}

Bastos, D. O. (2014). Estudo da circunferência no ensino médio: sugestões de atividades com a utilização do software GeoGebra. Dissertação de Mestrado. Mestrado Profissional em Matemática em Rede Nacional - PROFMAT, Universidade Federal do Rio Grande, Brasil.

Batanero, C., Burrill, G. e Reading, C. (2011). Teaching statistics in school mathematicschallenges for teaching and teacher education.ICMI/IASE study: The 18th ICMI Study.

Bishop, A. J., Clements, K., Keitel, C., Kilpatrick, J. E Leung, F. K. S. (2003). Second international handbook of mathematics education. Dordrecht, The Netherlands: Kluwer.

Breda, A. (2016). Melhorias no ensino de matemática na concepção de professores que realizam o mestrado profmat no rio grande do sul: uma análise dos trabalhos de conclusão de curso. Tese de Doutorado. Pontifícia Universidade Católica do Rio Grande do Sul, Porto Alegre, Brasil:

Breda, A., Font, V. e Lima, V. M. R. (2015). A noção de idoneidade didática e seu uso na formação de professores de matemática. Jornal Internacional de Estudos em Educação Matemática, 8(2), 1-41.

Breda, A. e Lima, V. M. R. (2016). Estudio de caso sobre el análisis didáctico realizado en un trabajo final de un máster para profesores de matemáticas en servicio. REDIMAT Journal of Research in Mathematics Education, 5, 74-103.

Breda, A., Pino-Fan, L. e Font, V. (2016). Establishing criteria for teachers' reflection on their own practices. In Csíkos, C., Rausch, A. \& Szitányi, J. (Eds.), Proceedings of the 40th Conference of the International Group for the Psychology of Mathematics Education (Vol. 1, pp-283). Szeged, Hungary: PME.

Cevallos, I. (2011). O mestrado profissional em ensino de matemática e o desenvolvimento profissional de professores: um desafio institucional. Tese de Doutorado. Pontifícia Universidade Católica de São Paulo, Brasil.

Coordenação de Aperfeiçoamento de Pessoal de Nível Superior (2013). Avaliação suplementar externa do programa de mestrado profissional em matemática em rede nacional (PROFMAT). Brasil: Autor. 
English, L. D., Bartolini-Busi, M., Jones, G. A., Lesh, R. e Tirosh, D. (2008). Handbook of international research in mathematics education. London: Lawrence Erlbaum.

Fauvel, J. e Maanen, J. (2000). The role of the history of mathematics in the teaching and learning of mathematics.Dordrecht, The Netherlands: Kluwer.

Ferreira, E. C. (2013). Práticas pedagógicas e objetos de estudo [manuscrito]: análise sobre as pesquisas em educação matemática do programa de mestrado da UEPB nos anos de 2007 e 2008. Dissertação de Mestrado em Ensino de Ciências e Matemática. Universidade Estadual da Paraíba, João Pessoa, Brasil.

Ferreres, S e Vanegas, Y. (2015). Uso de criterios de calidad en la reflexión sobre la práctica de los futuros profesores de secundaria de matemáticas. Procedia, 196(8), 219-225.

Font, V. (1999). Procediments per obtenir expressions simbòliques a partir de gràfiques. Aplicacions a les derivades. Tesis doctoral no publicada. Universitat de Barcelona, Barcelona.

Font, V. (2008). Enseñanza de las matemáticas. Tendencias y perspectivas. En C. Gaita (Ed.), Actas del III Coloquio Internacional sobre Enseñanza de las Matemáticas (21-62). Lima, Perú: Pontificia Universidad Católica del Perú.

Font, V. (2015). Pauta de análisis y valoración de la idoneidad didáctica de procesos de enseñanza y aprendizaje de la matemática. Documento no publicado. Departamento de Didáctica de las CCEE y la Matemática de la Universitat de Barcelona.

Font, V., Planas, N. e Godino, J. D. (2010). Modelo para el análisis didáctico en educación matemática. Infancia y Aprendizaje, 33(1), 89-105.

Giménez, J., Vanegas, Y., Font, V., Ferreres, S. (2012). El papel del trabajo final de Máster en la formación del profesorado de Matemáticas. UNO. Revista de Didàctica de las Matemáticas, 61, 76-86.

Godino, J. D. (2013). Indicadores de la idoneidad didáctica de procesos de enseñanza y aprendizaje de las matemáticas. Cuadernos de Investigación y Formación en Educación Matemática 11, 111-132.

Godino, J. D., Batanero, C. e Font, V. (2007). The onto-semiotic approach to research in mathematics education.ZDM. The International Journal on Mathematics Education, $39(1), 127-135$.

Godino, J. D., Bencomo, D., Font, V. e Wilhelmi, M. R. (2007). Pauta de análisis y valoración de la idoneidad didáctica de procesos de enseñanza y aprendizaje de la matemática. Recuperado de: http://www.ugr.es/ jgodino/funcionessemioticas/pauta_valoracion_idoneidad_5enero07.pdf

Godino, J. D., Contreras A. e Font, V. (2006). Análisis de procesos de instrucción basado en el enfoque ontológico-semiótico de la cognición matemática. Recherches en Didactique des Mathématiques, 26(1), 39-88.

Godino, J. D., Font, V., Wilhelmi, M. R. e Castro, C. (2009). Aproximación a la dimensión normativa en didáctica de las matemáticas desde un enfoque ontosemiótico. Enseñanza de las Ciencias, 27(1), 59-76.

Gonçalves, T. S. (2013). Uma introdução à geometria projetiva para o ensino fundamental. Dissertação de Mestrado. Mestrado Profissional em Matemática em Rede Nacional PROFMAT, Universidade Federal do Rio Grande, Brasil.

Gutiérrez, A. e Boero, P. (2006). Handbook of research on the psychology of mathematics education. Rotterdam, The Netherlands: Sense Publishers.

Guzmán, M. (2007). Enseñanza de las ciencias y la matemática. Revista Iberoamericana de Educación, 43, 19-58. 
Hanna, G. (1996). Towards gender equity in mathematics education. An ICMI Study. Dordrecth, The Netherlands: Kluwer Academic Publishers.

Holton, D. (2001). Teaching and learning of mathematics at university level. Dordrecht, The Netherlands: Kluwer.

Jung, M. O. (2013). Questionário virtual para o ensino de probabilidade. Dissertação de Mestrado. Mestrado Profissional em Matemática em Rede Nacional - PROFMAT, Universidade Federal do Rio Grande, Brasil.

Lester, F. (2007). Second handbook of research on mathematics teaching and learning. Greenwich, Connecticut: Information Age Publishing e NCTM.

Llinares, S. (2012). Construcción de conocimiento y desarrollo de una mirada profesional para la práctica de enseñar matemáticas en entornos en línea. Avances de Investigación en Educación Matemática 2, 53 - 70.

Lopes, A. (2014). Um relato sobre à introdução às somas de Riemann na educação básica. Dissertação de Mestrado. Mestrado Profissional em Matemática em Rede Nacional PROFMAT, Universidade Federal de Santa Maria, Brasil.

Ludke, M. e André, M. E. D. A. (1986). Pesquisa em educação: abordagens qualitativas. São Paulo, Editora Pedagógica e Universitária.

Mammana, C. e Villani, V. (1998). Perspectives on the teaching of geometry for the 21st century. Dordrecht, The Netherlands: Kluwer.

Martins, T. E. (2014). Equações de recorrência na educação básica. Dissertação de Mestrado Mestrado Profissional em Matemática em Rede Nacional - PROFMAT, Universidade Federal do Rio Grande, Brasil.

Ministério da Educação (2000). Parâmetros curriculares nacionais do ensino médio. Brasilia: $\mathrm{MEC} / \mathrm{SEF}$.

Ministério da Educação (2014). Lei No 13.005. Aprova o Plano Nacional de Educação (PNE) e dá outras providências. Ministério da Fazenda (MF); Ministério do Planejamento, Orçamento e Gestão (MP); Ministério da Educação (MEC), 25 de junho de 2014. Brasil: Dilma Rousseff.

Mohnsam, J. C. (2014). As contribuições de Arquimedes para o cálculo de áreas. Dissertação de Mestrado. Mestrado Profissional em Matemática em Rede Nacional - PROFMAT, Universidade Federal do Rio Grande, Brasil.

Molon, J. (2013). Cálculo no ensino médio: uma abordagem possivel e necessária com o auxílio do software GeoGebra. Dissertação de Mestrado. Mestrado Profissional em Matemática em Rede Nacional - PROFMAT, Universidade Federal de Santa Maria, Brasil.

Moreira, M. A. (2004). O mestrado (profissional) em ensino. RBPG. Revista Brasileira de Pós-Graduação, 1(1), 131-142.

Müller, I. (2000). Tendências atuais de educação matemática. UNOPAR Científica Ciências Humanas e Educação. 1(1), 133-144.

NCTM (2000). Principles and Standards for School Mathematics. Reston: VA and National Council of Teachers of Mathematics.

Pinz, C. R. F. (2013). Dígitos verificadores e detecção de erros. Dissertação de Mestrado. Mestrado Profissional em Matemática em Rede Nacional - PROFMAT, Universidade Federal do Rio Grande, Brasil.

Posadas, P. (2013). Evaluación de la idoneidad didáctica de una experiencia de enseñanza sobre ecuaciones de segundo grado en $3^{\circ}$ de educación secundaria obligatoria. Dissertação de mestrado. Universidad de Granada, Granada. 
Posadas, P. e Godino, J. D. (2014). Reflexión sobre la práctica docente como estrategia formativa para desarrollar el conocimiento didáctico-matemático. Departamento de Didáctica de la Matemática. Universidad de Granada. Recuperado de: http://www.ugr.es/ jgodino/fprofesores/Posadas_reflexion.pdf.

Prado, M. R. M., Silva, M. G. L. e Araujo, M. F. F. (2011). A formação pós-graduada em Ensino de Ciências Naturais e Matemática de docentes do IFRN: implicações na atuação docente. Trabalho apresentado no VIII ENPEC/ I Encuentro Iberoamericano de Investigación en Didáctica de las Ciências, Campinas, Brasil.

Rondero, C. e Font, V. (2015). Articulación de la complejidad matemática de la media aritmética. Enseñanza de las Ciencias, 33(2), 29-49.

Schäfer, E. D. A. (2013). Impacto do mestrado profissional em ensino de física da UFRGS na prática docente: um estudo de caso. Tese de Doutorado. Universidade Federal do Rio Grande do Sul, Porto Alegre.

Seckel, M. J. (2016). Competencia en análisis didáctico en la formación inicial de profesores de educación general básica con mención en matemática. Tese de Doutorado. Universitat de Barcelona, Barcelona, España.

Secretaria de Estado da educação do Paraná. (2008). Diretrizes curriculares de matemática para a educação básica. SEED: Curitiba.

Sociedade Brasileira de Matemática (2013). Uma análise quali-quantitativa de perfis de candidatos ao Mestrado Profissional em Matemática em Rede Nacional (PROFMAT). Relatório final do procedimento de análise quali-quantitativa de perfis de candidatos e aprovados no Mestrado Profissional em Matemática em Rede Nacional (PROFMAT). Brasil: Autor.

Souza, M. E. D. (2013). Uma proposta de abordagem ao problema de Flávio Josefo aplicada ao Ensino Médio. Dissertação de Mestrado. Mestrado Profissional em Matemática em Rede Nacional - PROFMAT, Universidade Federal de Santa Maria, Brasil.

Souza, S. A. (2014). Estudo do produto matricial por meio do Método dos Mínimos Quadrados: uma abordagem destinada ao Ensino Médio. Dissertação de Mestrado. Mestrado Profissional em Matemática em Rede Nacional - PROFMAT, Universidade Federal de Santa Maria, Brasil.

Strasburg, E. B. (2014). Atividades de trigonometria para o ensino fundamental com o uso do software GeoGebra. Dissertação de Mestrado. Mestrado Profissional em Matemática em Rede Nacional - PROFMAT, Universidade Federal do Rio Grande, Brasil.

Stacey, K., Chick, H. e Kendal, M. (Eds) (2004). The future of the teaching and learning of algebra: The 12th ICMI Study. Kluwer, Dordrecht.

\section{Referências dos autores}

Adriana Breda, Universidad de Los Lagos, (Chile), adriana.breda@gmail.com

Vicenç Font, Universitat de Barcelona, (Espanha), vfont@ub.edu

Valderez Marina do Rosário Lima, Pontifícia Universidade Católica do Rio Grande do Sul, (Brasil), valderez.lima@pucrs.br 


\title{
Analysis of innovation proposals from the final course assignments in a professional master program in mathematics
}

\author{
Adriana Breda, Universidad de Los Lagos, (Chile) \\ Vicenç Font, Universitat de Barcelona, (Espanha) \\ Valderez Marina do Rosário Lima, Pontifícia Universidade Católica do Rio Grande do \\ Sul, (Brasil)
}

The main objective of this work is finding out the innovations and their justifications within the Brazilian Professional Master Program in Mathematics (PROFMAT). To achieve this goal, a literature review was carried out by considering: i) the main tendencies in mathematics education were described as a guide for the implementation of processes related to quality mathematics teaching; iii) the different stages of the instructional process; iii) some studies that have focused on the analysis of final works on Science and Mathematics Education areas.

From the methodological point of view, a total of 29 final course assignments were selected for this study, which were presented at the University of Rio Grande do Sul state and published along the years 2013 and 2014. A qualitative analysis of these documents was developed, where all the works were classified according to the type of innovation proposed and to the phase of the instructional process taken into account. Moreover, we highlighted the arguments used by the authors to justify their innovative proposals.

It is concluded that the main kinds of innovation are (i) incorporation of higher education content on basic education and (ii) introduction of computational resources. The former is justified because it allows new ways of relating and addressing mathematical content, conducting relevant mathematical processes and doing more attractive the lessons to stimulate the students' interest. The latter is justified by the fact that ICT motivates and stimulate lessons that contribute to facilitate conceptual understanding and problem solving. 